\title{
Brucea javanica oil emulsion suppresses tumor growth in human cervical cancer cells through inhibition of the E6 oncogene and induction of apoptosis
}

\author{
Ling Ye ${ }^{1}$, Jian-Fu Zhao ${ }^{1}$, Yi-Ming Wang ${ }^{1}$, Wen-Hui Chen ${ }^{1}$, Shen Qian ${ }^{2}$, Zhong-Guo Zhou ${ }^{3}, M$ Mun $^{1}$ \\ ${ }^{1}$ Department of Oncology, The First Affiliated Hospital of Jinan University, Guangzhou 510632, China; ${ }^{2}$ The Third Department of Pulmonary \\ Disease, The Third People's Hospital of Shenzhen, Shenzhen 518112, China; ${ }^{3}$ Department of Liver Surgery, Sun Yat-sen University Cancer Center, \\ Guangzhou 510060, China \\ Contributions: (I) Conception and design: All authors; (II) Administrative support: L Ye, M Xu; (III) Provision of study materials or patients: L Ye, \\ JF Zhao, YM Wang, ZG Zhou; (IV) Collection and assembly of data: Ling Ye, Wen-Hui Chen, Shen Qian; (V) Data analysis and interpretation: \\ L Ye, JF Zhao, WH Chen; (VI) Manuscript writing: All authors; (VII) Final approval of manuscript: All authors. \\ Correspondence to: Shen Qian. The Third Department of Pulmonary Disease, The Third People's Hospital of Shenzhen, Shenzhen 518112, China. \\ Email: Qianshen7@aliyun.com; Zhong-Guo Zhou. Department of Liver Surgery, Sun Yat-sen University Cancer Center, Guangzhou 510060, China. \\ Email: zhouzhg@sysucc.org.cn; Meng Xu. Department of Oncology, The First Affiliated Hospital of Jinan University, Guangzhou 510632, China. \\ Email: 641704010@qq.com.
}

Background: Brucea javanica oil emulsion (BJOE) is a traditional Chinese medicine with recognized antitumor effects in various cancers, but the effects and mechanisms of action of BJOE against cervical cancer need to be further studied. Herein, we investigated the effects of BJOE on the human papillomavirus (HPV)16-expressing human cervical cancer line $\mathrm{SiHa}$ and explored the possible underlying mechanisms.

Methods: Cell viability and apoptosis of SiHa cells treated with BJOE were assessed by the MTT [3-(4,5-dimethylthiazol-2-yl)-2,5-diphenyl tetrazolium bromide] and annexin V-fluorescein isothiocyanate (annexin V-FITC)/propidium iodide (PI) staining assays, respectively. Quantitative reverse transcriptionpolymerase chain reaction and Western blot analyses were performed to assess the expression levels of the E6 oncogene and key signaling molecules involved in apoptosis. A subcutaneous xenograft nude mouse model bearing SiHa cells was established and treated with BJOE through intraperitoneal injection. Tumor growth was monitored, and immunohistochemical analysis was performed.

Results: BJOE exhibited substantial cytotoxic effects in SiHa cells and significantly suppressed tumor growth in SiHa cell xenografts. BJOE inhibited E6 expression and induced apoptosis in vitro in a dosedependent manner. BJOE-induced apoptosis was characterized by activation of caspase-3 and cleavage of poly(ADP-ribose) polymerase (PARP). Moreover, BJOE induced phosphorylation of extracellular-signal regulated kinase (ERK) and inhibited the expression of nuclear factor-kappa B (NF- $\kappa$ B).

Conclusions: BJOE exerts a strong tumor-suppressive effect in $\mathrm{SiHa}$ cells in vitro and in vivo, likely caused by E6 inhibition and apoptosis induction achieved through the ERK/mitogen-activated protein kinase (MAPK) and NF- $\mathrm{KB}$ signaling pathways, supporting potential use of BJOE in cervical cancer treatment.

Keywords: Brucea javanica oil; cervical cancer; apoptosis; human papillomavirus (HPV); tumor suppression

Submitted Aug 04, 2019. Accepted for publication Nov 29, 2019.

doi: $10.21037 /$ tcr.2019.12.62

View this article at: http://dx.doi.org/10.21037/tcr.2019.12.62 


\section{Introduction}

Cervical cancer is a major global public health problem, ranking as the fourth most frequently diagnosed malignancy and cause of cancer-related death in women (1). In China, an estimated 106,430 new cases of cervical cancer and 47,739 deaths were expected in 2018, accounting for about $20 \%$ of global cases (2). A remarkable disparity in the incidence and mortality from cervical cancer between lowand middle-income countries (LMIC) and high-income countries (HIC) has been observed, with about $85 \%$ of new cases and $90 \%$ of deaths occurring in LMIC (1). Much of this disparity is attributable to substantial differences in prevention and treatment strategies.

Virtually all cervical cancer cases are caused by persistent infection of human papillomavirus (HPV), with the oncogenic HPV16 and 18 being the most prevalent types in cervical cancer (3). Consequently, prophylactic HPV vaccination against HPV16/18 of adolescent girls has been introduced in many countries since 2006 to prevent cervical cancer (4). This HPV vaccination strategy, along with cervical cancer screening to identify precancerous lesions, has been proven to reduce and potentially eliminate the burden of cervical cancer in HIC effectively; yet in LMIC, the access to HPV vaccination and cancer screening services has been limited $(5,6)$. The HPV vaccine was licensed in 2016 in China; although various efforts have been made, high costs and inadequate coverage form barriers to the widespread use of HPV vaccines, especially in less developed areas (7). Consequently, cervical cancer remains a common disease with significant morbidity in Chinese women.

HPVs infect the epithelial cells of the mucosa and skin and are categorized as low-risk or high-risk, depending on their oncogenicity. HPV16 is the predominant oncogenic strain identified in cervical precancerous and cancerous lesions. It is well recognized that after integration into the host genome, the oncogenic activities of HPVs are largely attributed to the viral E6 and E7 oncoproteins, which act synergistically or independently to immortalize and malignantly transform the host cells by inactivating the tumor suppressors $\mathrm{p} 53$ and retinoblastoma protein $(\mathrm{pRb})$, respectively, thus leading to genomic instability, aberrant apoptosis, and uncontrolled proliferation (8). Continuous expression of E6 and E7 is critical to maintain the malignant phenotype of HPV-positive cancer cells (9). The expression of E6 and E7 oncoproteins has been found to alter multiple signaling pathways, among which the phosphoinositide 3-kinase (PI3K)/AKT/mammalian target of rapamycin (mTOR), Wnt, Notch, and Ras/Raf/mitogen- activated protein kinase (MAPK)/extracellular-signal regulated kinase (ERK) signaling pathways already have been studied as therapeutic targets in HPV-positive cervical cancer. Targeted manipulation of the pathways that mediate the effects of E6 and E7 represent a promising approach for the treatment of cervical cancer.

Brucea javanica oil emulsion (BJOE), extracted from the dry and ripe fruit of Brucea javanica with purified soybean lecithin as an emulsifier, is a traditional Chinese medicine product that has been used to treat chronic gastritis, colitis, and cancers in China for centuries. BJOE has demonstrated a favorable effect on radiotoxicity and quality of life in patients receiving radiotherapy for advanced esophageal cancer (10). Accumulating clinical studies indicate that BJOE combined with conventional chemotherapy could provide more beneficial effects than chemotherapy alone for patients with various types of cancer, including lung, gastric, and liver cancers (11-15). BJOE has been found to induce cytotoxicity in HPV-positive cervical cancer cells selectively by targeting the apoptosis mechanism (16), suggesting its therapeutic potential for cervical cancer. However, the amount of evidence is limited and the underlying mechanisms have not been fully elucidated.

This study evaluated the in vitro effects of BJOE on the viability and apoptosis of HPV16-expressing cervical cancer cells as well as investigated whether BJOE can suppress tumor growth in an in vivo xenograft model. In addition, we elucidated the mechanisms by which BJOE suppresses cervical cancer cell growth. Specifically, we targeted genes that are involved in apoptosis and inflammation.

\section{Methods}

\section{Cell culture}

The HPV16-expressing cervical carcinoma cell line SiHa was obtained from the American Type Culture Collection and cultured according to standard procedures. Briefly, the cells were cultured in Dulbecco's modified Eagle's medium (DMEM; Gibco) supplemented with $2 \mathrm{mM}$ L-glutamine and $10 \%$ fetal bovine serum (Gibco) and maintained in a humidified atmosphere containing $5 \% \mathrm{CO}_{2}$ at $37^{\circ} \mathrm{C}$.

\section{Cell viability assay}

The inhibitory effects of BJOE against SiHa cells were measured using the MTT cell viability assay. To assess the dose effect, $\mathrm{SiHa}$ cells were seeded on 24-well culture plates at a density of $1 \times 10^{6}$ cells $/ \mathrm{mL}$ and were treated with 0 (as a control), $62.5,125$, or $250 \mu \mathrm{g} / \mathrm{mL}$ BJOE extract solution 
for $24 \mathrm{~h}$. To assess the time effect, cells $\left(1 \times 10^{6}\right.$ cells $\left./ \mathrm{mL}\right)$ were treated with $250 \mu \mathrm{g} / \mathrm{mL} \mathrm{BJOE}$ for 0 (as a control), $3,6,12$, or $24 \mathrm{~h}$ in $5 \% \mathrm{CO}_{2}$ at $37^{\circ} \mathrm{C}$. Afterwards, the cells were harvested, washed twice with phosphate-buffered saline (PBS), and resuspended in PBS to a concentration of $1 \times 10^{6}$ cells $/ \mathrm{mL}$. The resuspended cells were seeded on 96well plates and incubated for $42 \mathrm{~h}$ before $20 \mu \mathrm{L}$ of MTT solution ( $5 \mathrm{mg} / \mathrm{mL}$; Sigma Aldrich) was added to each well. Then the cells were incubated for another $4 \mathrm{~h}$. After removal of the MTT [3-(4,5-dimethylthiazol-2-yl)-2,5diphenyl tetrazolium bromide]-containing medium, the cells were lysed with $150 \mu \mathrm{L}$ of dimethyl sulfoxide (Sigma) under shaking for $10 \mathrm{~min}$. The absorbance was measured at an optical density (OD) of $490 \mathrm{~nm}$ using a microplate reader (Thermo Scientific, USA). Samples were measured in ten replicates, and each experiment was repeated three times. The percentage of cell inhibition was calculated using the following formula: inhibitory rate $(\%)=(1-$ OD490 of the experimental sample)/OD490 of the control sample $\times 100$.

\section{Apoptosis assay}

The apoptotic effect of BJOE was measured using the annexin V-fluorescein isothiocyanate (annexin V-FITC)/ propidium iodide (PI) apoptosis detection kit (Kaiji Biotechnology, Nanjing, China), according to the manufacturer's recommendations. After treatment with various doses of BJOE $(0,62.5,125$, or $250 \mu \mathrm{g} / \mathrm{mL})$ for $24 \mathrm{~h}$, the adherent cells were detached by trypsin (Gibco) and harvested by centrifugation at $1,000 \mathrm{~g}$ for $5 \mathrm{~min}$. After being washed with PBS and resuspended in $400 \mu \mathrm{L}$ of $1 \times$ binding buffer, the cells were stained with $5 \mu \mathrm{L}$ of annexin V-FITC for $15 \mathrm{~min}$ and $10 \mu \mathrm{L}$ of ice-cold PI for $5 \mathrm{~min}$, in sequence, at room temperature in the dark. Flow cytometric analysis was performed with an excitation wavelength of $488 \mathrm{~nm}$ and an emission wavelength of $530 \mathrm{~nm}$ for annexin V-FITC and $575 \mathrm{~nm}$ for PI. Apoptotic cells were defined as positive for annexin V-FITC (green fluorescence) and negative for PI (red fluorescence), whereas necrotic cells were positive for both types of staining.

\section{Quantitative reverse transcription-polymerase chain reaction ( $q R T-P C R)$}

After BJOE treatment for $24 \mathrm{~h}$, the cells were washed with PBS, detached with trypsin, and harvested by centrifugation. Total cellular RNA was extracted with the TRIzol-based total RNA extraction kit (Promega), according to the manufacturer's instructions. For cDNA synthesis, total RNA was reverse transcribed using a PrimeScript RT Reagent kit with gDNA eraser (Takara Bio, USA). HPV16 E6 mRNA expression was measured by qRT-PCR using the SYBR Green master mix (Biotool) on a BioRad real-time PCR detection system. A calibration curve was obtained by serial dilution of the cDNA to optimize the amplification protocol. The relative expression level of E6 mRNA was normalized to that of the housekeeping gene $\beta$-actin and generated using the comparative cycle threshold method. Samples were measured in four replicates, and each experiment was repeated four times.

\section{Western blot analysis}

Cells were harvested after BJOE treatment, and total protein was extracted using standard cell lysis buffer (BGI Tech, Shenzhen, China). Total protein was measured using a bicinchoninic acid kit (Thermo Scientific), according to the manufacturer's instructions. Cell lysates containing equal amounts of total protein were loaded for sodium dodecyl sulfate-polyacrylamide gel electrophoresis. The separated proteins were transferred onto a polyvinylidene fluoride (PVDF) membrane using the wet transfer method. The PVDF membrane was then blocked with blocking buffer containing $5 \%$ dry milk and $0.1 \%$ Tween-20 in Trisbuffered saline and incubated overnight at $4{ }^{\circ} \mathrm{C}$ with one of the following primary antibodies: caspase-3, cleaved caspase-3, poly(ADP-ribose) polymerase (PARP), ERK, and phospho-ERK (p-ERK), nuclear factor-kappa B (NF-кB), and p53 (Cell Signaling Technology, USA). After incubation with the secondary antibody horseradish peroxidase (HRP)labeled goat anti-rabbit or goat anti-mouse immunoglobulin G (IgG, 1:8,000, Santa Cruz Biotechnology, USA) for $1 \mathrm{~h}$, the bound antibodies were visualized by chemiluminescence on radiographic films, and the blot density was quantified using Image $\mathrm{J}$ software.

\section{Subcutaneous xenograft model}

A subcutaneous xenograft model of human cervical cancer was established by subcutaneous injection of $\mathrm{SiHa}$ cells into the back of 6-8-week-old nude mice. The tumors were allowed to grow for 2 weeks. The nude mice were then randomly assigned into the experimental or control group ( $\mathrm{n}=10$ for each group), receiving an intraperitoneal injection of BJOE extract $(50 \mathrm{mg} / \mathrm{kg} / \mathrm{d}$ ) or $0.9 \%$ saline, respectively, for 30 continuous days. The tumor size was monitored every 3 days and was calculated using the following formula: 
tumor volume $=\left(\right.$ length $\times$ width $\left.^{2}\right) / 2$. Three days after the last injection, the mice were sacrificed and the tumor tissues were harvested. All animal experiments were conducted in accordance with the National Institutes of Health Guide for the Care and Use of Laboratory Animals, and the animal protocol was approved by the Ethics Committee of Jinan University. The nude mice were purchased from the Experimental Animal Center of Jinan University and were maintained under specific pathogen-free conditions.

\section{Tumor histology and immunohistochemistry (IHC)}

The harvested tissues were fixed in $4 \%$ paraformaldehyde, embedded, and sectioned for hematoxylin and eosin (H\&E) staining or IHC analysis. H\&E staining was performed using a standard procedure. For IHC analysis, tumor sections were deparaffinized by xylene, rehydrated through gradient ethanol to PBS, and permeabilized by microwave irradiation before being subjected to antigen retrieval. After incubation in 3\% hydrogen peroxide for $20 \mathrm{~min}$ to block endogenous peroxidases and washing with PBS, the sections were blocked with $10 \%$ normal goat serum at room temperature for $30 \mathrm{~min}$ and then incubated with primary antibody overnight at $4{ }^{\circ} \mathrm{C}$. After washing with PBS, the sections were incubated with the corresponding HRP-labeled secondary antibody (goat-anti-rabbit or goat-anti-mouse $\operatorname{IgG}$ ) at room temperature for $30 \mathrm{~min}$. After washing with PBS, the sections were visualized with diaminobenzidine and counterstained with hematoxylin. Three areas were randomly selected from each section, and positive staining was quantified in terms of the integrated OD using Image-Pro Plus 5.0 software.

\section{Statistical analysis}

Data are expressed as the mean \pm standard deviation (SD). The chi-squared test was used for comparing categorial data. One-way analysis of variance followed by Duncan's multiple range test was used for comparing measurements between groups. A P value $<0.05$ was considered statistically significant. All tests were performed using SPSS statistical software, version 17.0 (SPSS Inc., Chicago, IL, USA).

\section{Results}

BFOE suppresses cell viability and induces apoptosis in SiHa cells in vitro

To evaluate the cytotoxic effects of BJOE, we analyzed the cell viability and apoptotic cell populations (Figure 1). As shown in Figure 1A, BJOE inhibited the proliferation of $\mathrm{SiHa}$ cells in a dose-dependent manner. The cell viability was significantly decreased by $52.3 \%$ in $\mathrm{SiHa}$ cells treated with $250 \mu \mathrm{g} / \mathrm{mL}$ BJOE for $24 \mathrm{~h}$, compared with untreated cells. In SiHa cells treated with $250 \mu \mathrm{g} / \mathrm{mL}$ BJOE, the inhibitory effect of BJOE on cell viability was timedependent, with the decrease more pronounced after $12 \mathrm{~h}$ (Figure 1B). Annexin V-FITC/PI staining is generally used to distinguish apoptotic and necrotic cells from normal live cells. As shown in Figure 1C,D, BJOE increased the apoptotic and necrotic cell population in a dose-dependent manner, with a greater than 3.5 -fold increase in the number of apoptotic cells among cells treated with $250 \mu \mathrm{g} / \mathrm{mL}$ BJOE for $24 \mathrm{~h}$, compared with untreated cells. These results clearly indicate that BJOE inhibited the proliferation and induced the apoptosis of SiHa cells. The effects were dose-dependent, and the lowest dose tested $(62.5 \mu \mathrm{g} / \mathrm{mL})$ appeared to be effective for induction of apoptosis.

\section{BJOE inhibits $m R N A$ expression of E6 and recovers $p 53$ expression in SiHa cells}

The most prominent function of the viral E6 oncoprotein is to bind, ubiquitinate, and degrade $\mathrm{p} 53$, which is a critical tumor suppressor that regulates growth arrest and apoptosis, depending on the severity of DNA damage (9). To evaluate the effects of BJOE on E6-mediated p53 inactivation, we measured the expression of E6 and p53 in $\mathrm{SiHa}$ cells treated with various doses of BJOE by qRT-PCR (Figure 2) and Western blot analysis (Figure $3 A, B$ ), respectively. A significant dose-dependent decrease was observed in E6 expression at the transcriptional level; meanwhile, p53 protein expression was significantly increased in BJOEtreated cells compared with untreated cells, with the most significant increase of $\mathrm{p} 53$ expression in cells treated with a high dose of BJOE $(250 \mu \mathrm{g} / \mathrm{mL})$. These results indicate that the activity of $\mathrm{p} 53$ in HPV16-expressing SiHa cells was restored by BJOE treatment.

\section{BJOE suppresses the protein expression of $\mathrm{NF}-\mathrm{\kappa} B$ in SiHa cells}

The NF- $\mathrm{KB}$ signaling pathway is a key mediator of inflammatory and immune responses to some viral infections. In addition, NF- $\mathrm{\kappa B}$ activation triggered by the HPV16 E6 oncoprotein is known to cause abrogation of apoptosis (17). Moreover, constitutive NF- $\mathrm{NB}$ activation has been reported in cervical intraepithelial neoplasia and 
A

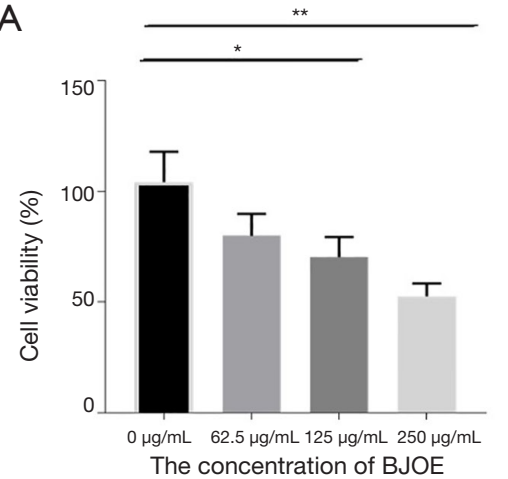

C

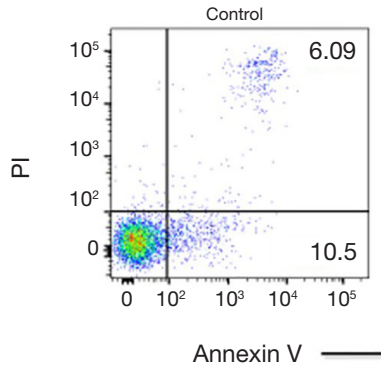

B
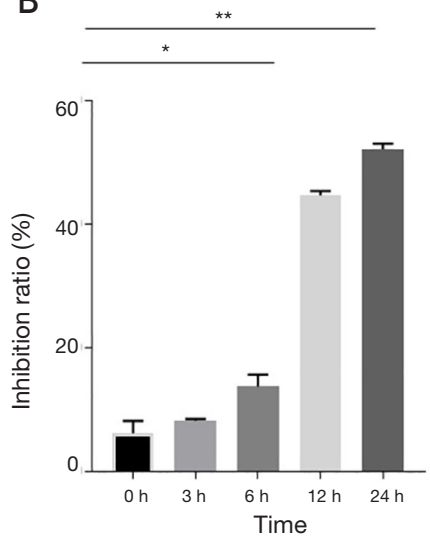

$\mathrm{D}$

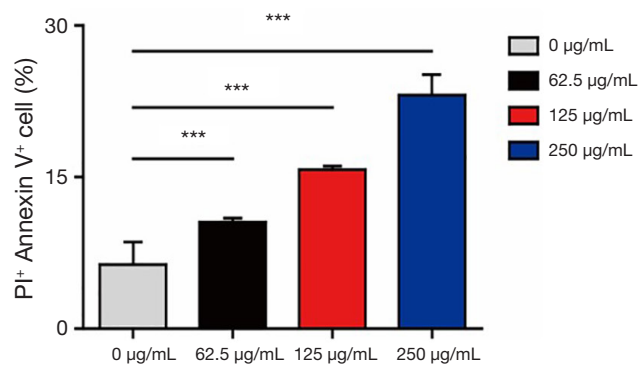

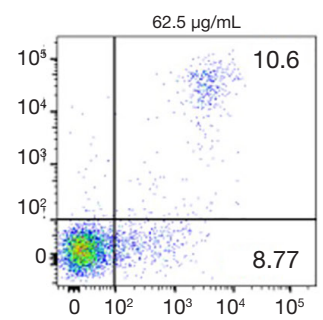
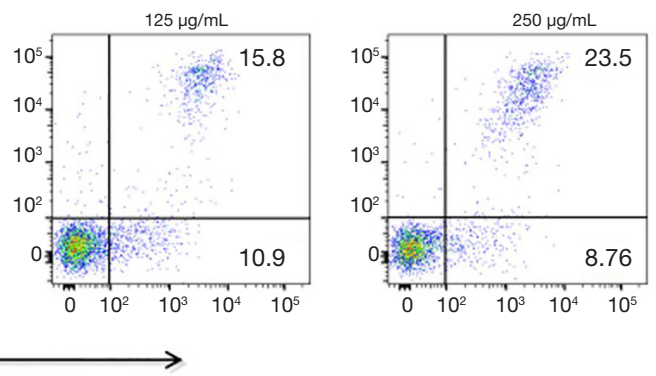

Figure 1 Effects of BJOE on the viability and apoptosis of SiHa cells. (A) The percentage of cell viability as determined by the MTT assay. $\mathrm{SiHa}$ cells were treated with various doses $(0,62.5,125$, and $250 \mu \mathrm{g} / \mathrm{mL})$ of BJOE for $24 \mathrm{~h}$. Mean $\pm \mathrm{SD},{ }^{*}, \mathrm{P}<0.05 ;{ }^{* *}, \mathrm{P}<0.01$, compared with $0 \mu \mathrm{g} / \mathrm{mL}$ BJOE at $24 \mathrm{~h}$. (B) Inhibitory rates of SiHa cells as determined by the MTT assay. SiHa cells were treated with $62.5 \mu \mathrm{g} / \mathrm{mL}$ BJOE for various time periods $(0,3,6,12$, and $24 \mathrm{~h})$. Mean $\pm \mathrm{SD},{ }^{*}, \mathrm{P}<0.05$; ${ }^{* *}, \mathrm{P}<0.01$, compared with BJOE treatment at baseline $(0 \mathrm{~h})$. (C) Representative cytometric dot plots showing annexin V-FITC and PI staining of SiHa cells treated with various doses of BJOE for $24 \mathrm{~h}$. (D) Quantification of the percentage of apoptotic cells treated with various doses of BJOE for $24 \mathrm{~h}$. Mean $\pm \mathrm{SD},{ }^{* * *}, \mathrm{P}<0.001$, compared with $0 \mu \mathrm{g} / \mathrm{mL}$ BJOE at $24 \mathrm{~h}$. BOJE, Brucea javanica oil emulsion; FITC, fluorescein isothiocyanate; PI, propidium iodide.

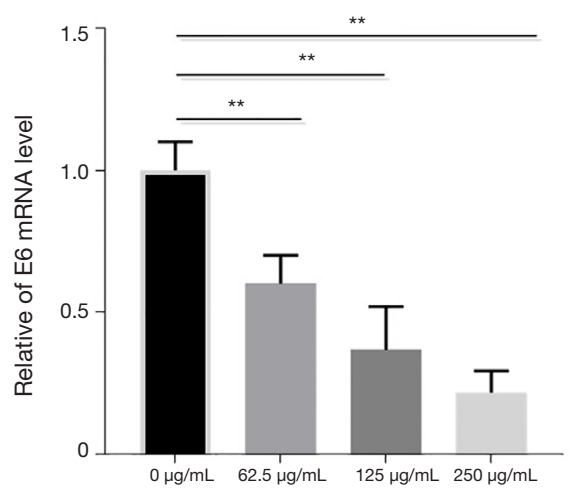

Figure 2 Relative expression of E6 mRNA in SiHa cells treated with various doses $(0,62.5,125$, and $250 \mu \mathrm{g} / \mathrm{mL})$ of BJOE for $24 \mathrm{~h}$. The relative expression level of E6 mRNA was determined by qRT-PCR. Mean $\pm \mathrm{SD},{ }^{* *}, \mathrm{P}<0.01$, compared with no treatment. BOJE, Brucea javanica oil emulsion; SD, standard deviation. cancer, and it has been associated with tumor invasion, progression, and metastasis (18). In this study, NF- $\kappa \mathrm{B}$ expression in response to BJOE treatment was assessed by Western blot analysis. As shown in Figure 3C, NF-кB expression was significantly decreased in a dose-dependent manner upon BJOE treatment. This result indicates that $\mathrm{NF}-\kappa \mathrm{B}$ activation in $\mathrm{SiHa}$ cells was reversed by BJOE treatment.

\section{BfOE activates caspase- 3 and cleaves PARP in SiHa cells}

Apoptosis is commonly mediated by the sequential activation of caspases, which execute apoptosis through the cleavage of several key proteins necessary for cell survival (19). Cleavage of PARP by caspase-3 is generally considered a hallmark of apoptosis, so we evaluated the 
A
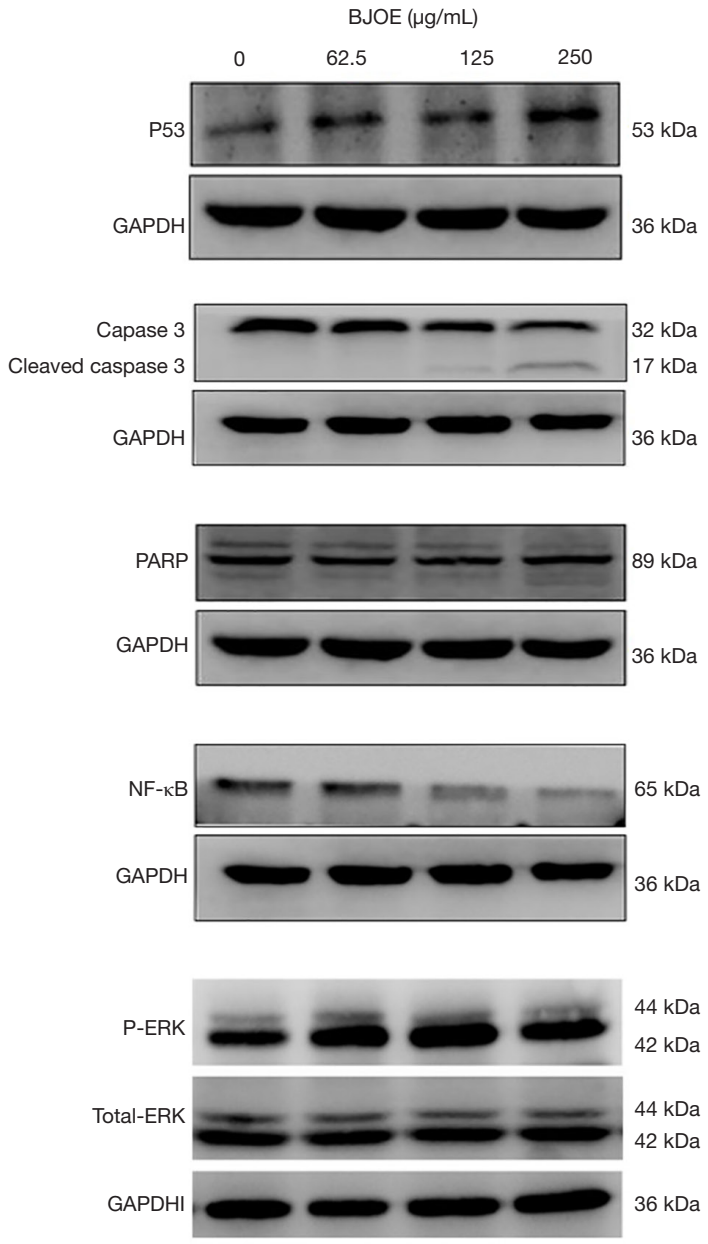

$\mathrm{H}$

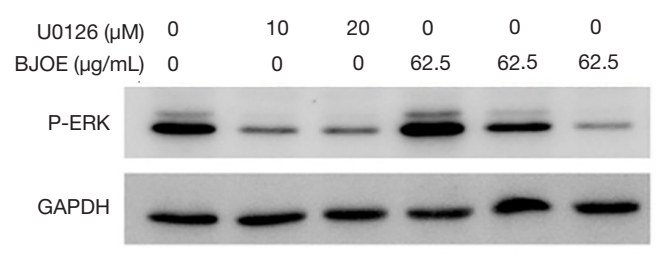

B

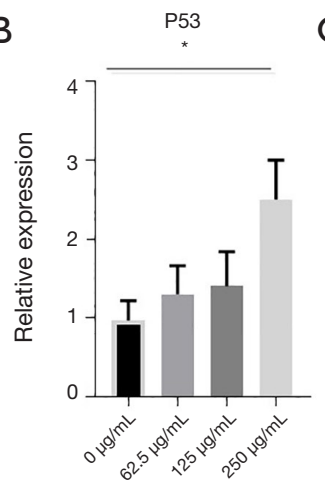

C

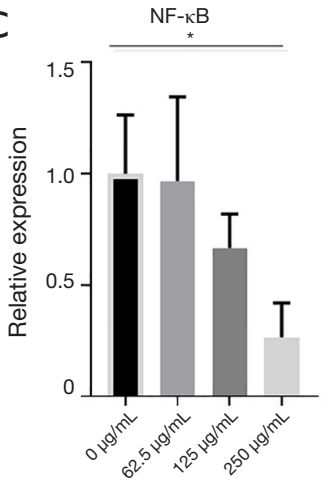

D

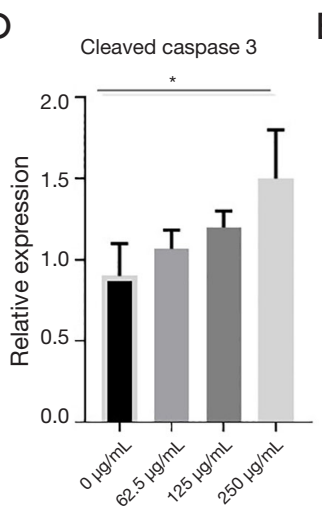

E
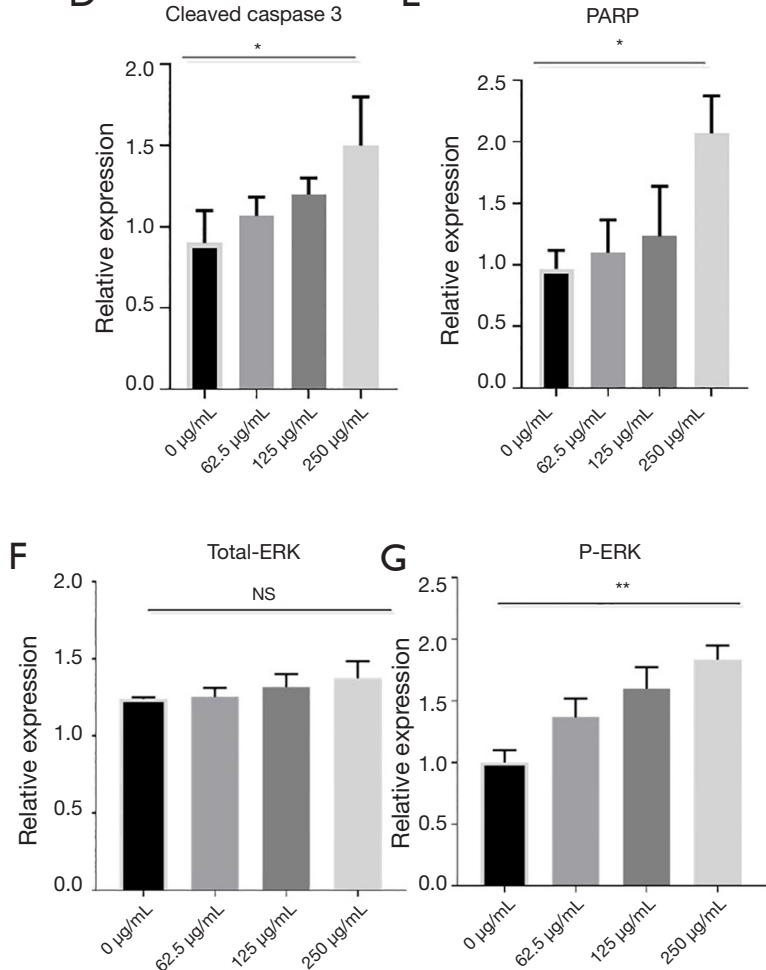

Figure 3 Effects of BJOE on the protein levels of apoptosis-related genes in SiHa cells. (A) Western blot images of the caspase-3, cleaved caspase-3, PARP, NF-kB, p53, ERK, and p-ERK proteins in SiHa cells treated with various doses $(0,62.5,125$, and $250 \mu \mathrm{g} / \mathrm{mL})$ of BJOE for 24 h. (B,C,D,E,F,G) Densitometric analysis of Western blot images for the relative expression of each protein in SiHa cells treated with various doses of BJOE for $24 \mathrm{~h}$. Mean $\pm \mathrm{SD}, \mathrm{NS}=$ no significant difference. ${ }^{*}, \mathrm{P}<0.05 ;{ }^{* *}, \mathrm{P}<0.01$, compared with $0 \mu \mathrm{gg} / \mathrm{mL}$ BJOE at $24 \mathrm{~h}$. (H) Western blot images of the p-ERK protein in SiHa cells treated with various doses $(0,10$, and $20 \mu \mathrm{M})$ of U0126, with and without BJOE treatment for $24 \mathrm{~h}$. BOJE, Brucea javanica oil emulsion; PARP, poly(ADP-ribose) polymerase; NF-kB, nuclear factor-kappa B; ERK, extracellular-signal regulated kinase; p-ERK, phospho-ERK; SD, standard deviation. 


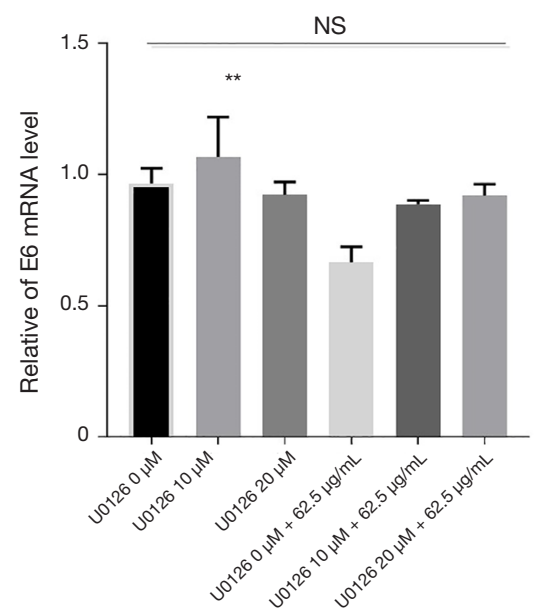

Figure 4 Relative expression of E6 mRNA in SiHa cells treated with various doses $(0,10$, and $20 \mu \mathrm{M})$ of $\mathrm{U} 0126$, with and without BJOE treatment for $24 \mathrm{~h}$. The relative expression level of E6 mRNA was determined by qRT-PCR. Mean $\pm \mathrm{SD}, \mathrm{NS}=$ no significant difference. ${ }^{* *}, \mathrm{P}<0.01$, compared with no treatment. BOJE, Brucea javanica oil emulsion; SD, standard deviation.

activation status of caspase- 3 and the expression of cleaved PARP by Western blot analysis (Figure 3D,E). Caspase-3 activation is achieved by proteolytic cleavage of the inactive $32-\mathrm{kDa}$ proenzyme into an active $17-\mathrm{kDa}$ subunit (Figure 3A). As shown in Figure 3D, BJOE induced the dose-dependent activation of caspase- 3 , which led to the cleavage of PARP. These results clearly indicate that BJOE can induce apoptosis via a caspase-3-dependent mechanism in $\mathrm{SiHa}$ cells.

\section{BJOE induces phosphorylation of ERK in SiHa cells}

The ERK/mitogen-activated protein kinase (MAPK) signaling pathway is well known for its role in apoptosis, and activation of ERK1/2 has been implicated in p53mediated apoptosis in cervical cancer cells (20). Due to the complexity of the ERK/MAPK signaling pathway, both antiapoptotic and proapoptotic functions of ERK1/2 have been reported in response to a wide range of stimuli and by transcriptionally regulating the activity of antiand proapoptotic molecules (21). Since BJOE inhibited E6 expression and activated p53 expression in $\mathrm{SiHa}$ cells, we explored whether BJOE modulates ERK activity by Western blot analysis. As shown in Figure $3 F, G$, the expression of total ERK was not affected by BJOE treatment, but a dose-dependent increase of phosphorylated
ERK, an active state of ERK, was observed. Next, we investigated the involvement of the ERK/MAPK signaling pathway in E6-mediated apoptosis by inhibiting the upstream kinase mitogen-activated protein kinase kinase (MEK)1/2 activity through its specific and potent inhibitor, U0126. The increased phosphorylation of ERK induced by BJOE treatment was substantially inhibited by U0126, in a dose-dependent manner (Figure 3H), but U0126 did not significantly alter the mRNA expression of E6 (Figure 4). The phosphorylated ERK levels were significantly greater in BJOE-treated cells as compared to untreated cells after exposure to $10 \mu \mathrm{M}$ U0126, indicating that an ERKdependent mechanism mediates BJOE-induced apoptosis.

\section{Intraperitoneal injection of BFOE inbibits subcutaneous cervical cancer xenograft growth}

To further evaluate the tumor-suppressive effects of BJOE in vivo, we established a nude mouse model harboring $\mathrm{SiHa}$ cell xenografts. SiHa cell xenografts were found to develop in $80 \%$ of the grafted mice. In the control mice injected with $0.9 \%$ saline, the tumors grew significantly faster and larger as compared with the BJOE-treated mice (Figure $5 A, B)$. As a result, the mean tumor weight in mice injected with BJOE was significantly reduced to less than half of that of the tumors in the control mice by 30 days after BJOE injection (Figure 5C). Consistent with the in vitro results, IHC analysis of the tumor tissues revealed that the proteins $\mathrm{p} 53$ and phosphorylated ERK were significantly expressed in the BJOE-treated mice as compared with the untreated mice (Figure 6). Taken together, these results support that BJOE treatment suppresses cervical cancer growth in vivo, which is achieved partly through induction of p53- and ERKmediated apoptosis.

\section{Discussion}

Suppression of apoptosis is central to carcinogenesis; therefore, targeting apoptosis is a viable and promising therapeutic option for cancer treatment. Most chemotherapeutic agents act as apoptosis inducers to inhibit the growth of cancer cells, but chemoresistance remains a major challenge in cancer therapy. Acquired resistance to apoptosis is a major mechanism allowing cancer cells to evade conventional chemotherapies, and a variety of molecular mechanisms have been implicated in the development of resistance to apoptosis in cancer cells $(19,22,23)$. Over the last few decades, considerable advances 


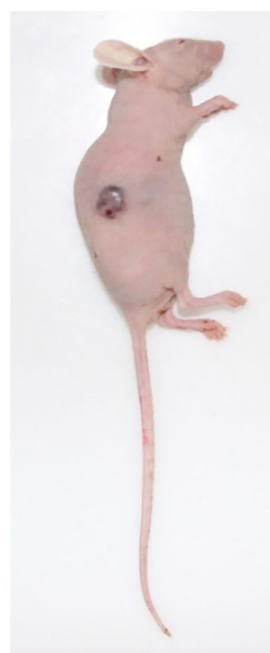

A
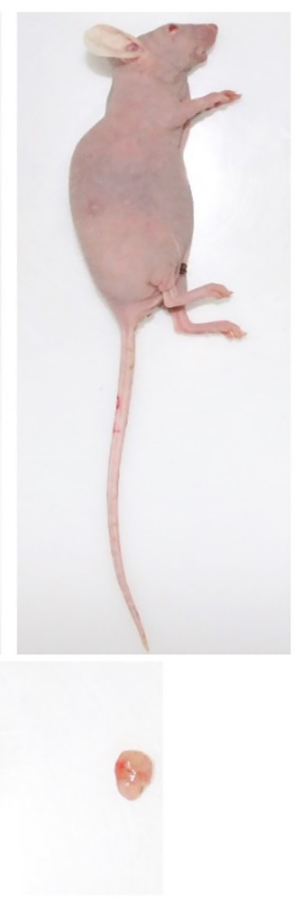

B
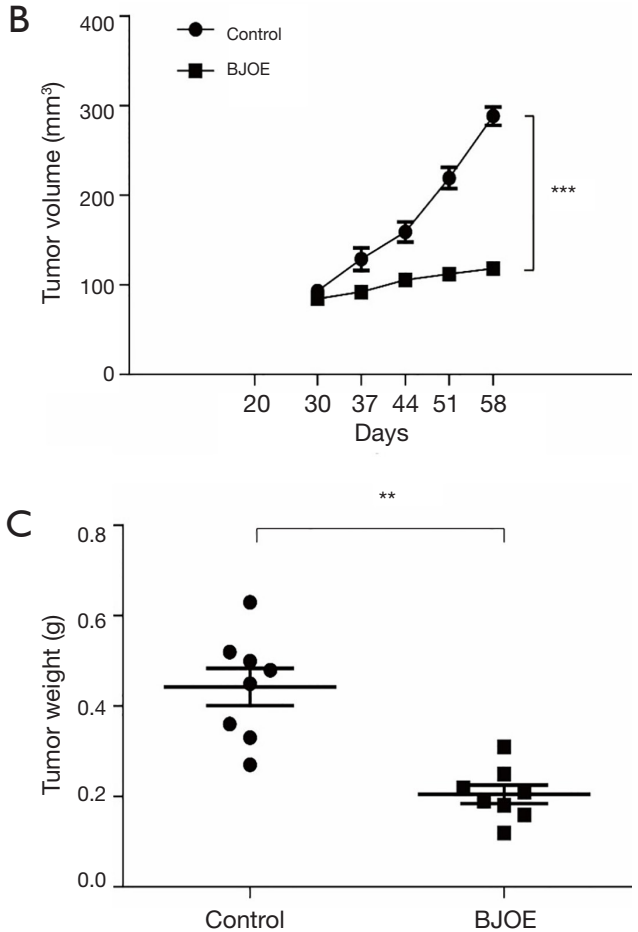

Figure 5 Effects of BJOE on tumor growth in the xenograft nude mouse model. (A) Images of nude mice with subcutaneous tumors and the corresponding tumor tissues. (B) The trends of tumor growth in terms of the tumor volume and (C) the tumor weight in the xenograft nude mouse model bearing SiHa cells after treatment with and without BJOE for 30 days. Mean $\pm \mathrm{SD}$. ${ }^{* *}, \mathrm{P}<0.01 ;{ }^{* * *}, \mathrm{P}<0.001$, compared with the control group. BOJE, Brucea javanica oil emulsion; SD, standard deviation.

have been made to develop novel therapeutic agents to prime and reactivate the apoptotic machinery in cancers resistant to conventional therapies (19). BJOE, a traditional Chinese medicine mainly comprised of oleic acid and linoleic acid, has been reported to exert a broad spectrum of antitumor activities by interfering with pathways involved in apoptosis, cell-cycle control, and angiogenesis $(24,25)$. Intriguingly, the clinical use of BJOE in combination with conventional chemotherapy has improved the efficacy without increasing complications in some cancers, suggesting a synergistic effect of BJOE with some chemotherapeutic agents (11-15). But no clinical application in cervical cancer has previously been reported, largely due to limited evidence of BJOE effectiveness in cervical carcinogenesis. Our results provide compelling evidence that $\mathrm{BJOE}$ is capable of inhibiting cervical tumor growth in vitro and in vivo. The mechanism underlying the tumor-suppressive effects of BJOE in $\mathrm{SiHa}$ cells involves, at least partly, induction of apoptosis that is blocked by HPV16 E6 expression. The apoptotic effect of BJOE observed in cervical cancer cells in this study has been reported in other cancer types (25-29).

$\mathrm{SiHa}$ cells contain the integrated HPV16 genome, which encodes three oncogenes (E5, E6, and E7) that can transform normal cells into cancer cells when expressed independently. The HPV16 E6 oncoprotein has welldescribed roles in cervical carcinogenesis by interfering with cell-cycle control and apoptosis through controlling p53 degradation (30). Inactivation of $\mathrm{p} 53$ by E6 is transient; and reduction of E6 expression in cervical cancer cells results in reactivation of $\mathrm{p} 53$ and restoration of apoptosis (31). Our results are supportive of this mechanism, showing that E6 expression was reduced by BJOE treatment in a doseand time-dependent manner in $\mathrm{SiHa}$ cells. Consequently, reactivation of $\mathrm{p} 53$ induced by BJOE treatment led to suppressed cell viability and enhanced apoptosis in $\mathrm{SiHa}$ cells; in addition, it inhibited tumor growth in $\mathrm{SiHa}$ cell xenografts. These results suggest an antagonistic role for $\mathrm{BJOE}$ in HPV-induced cervical carcinogenesis through targeting the $\mathrm{E} 6$ oncogene in vitro and in vivo.

Protein kinase activities are critical for eukaryotic cells in response to a variety of extracellular stress conditions, including viral infection. ERK1/2 and NF- $\mathrm{BB}$ are critical signaling transduction molecules that are involved in cellular response to stress conditions and have been 


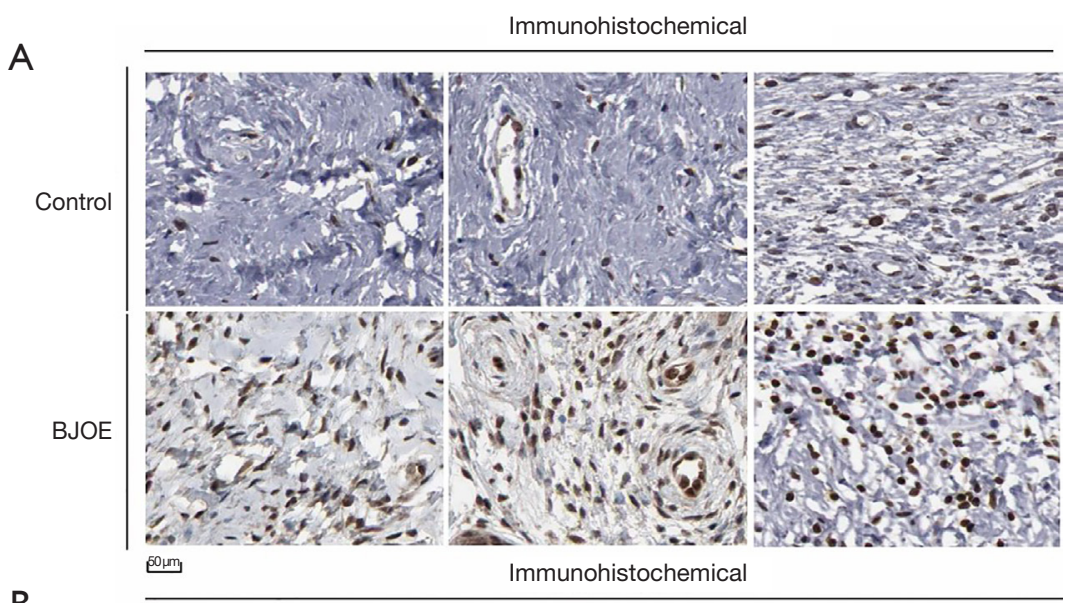

B

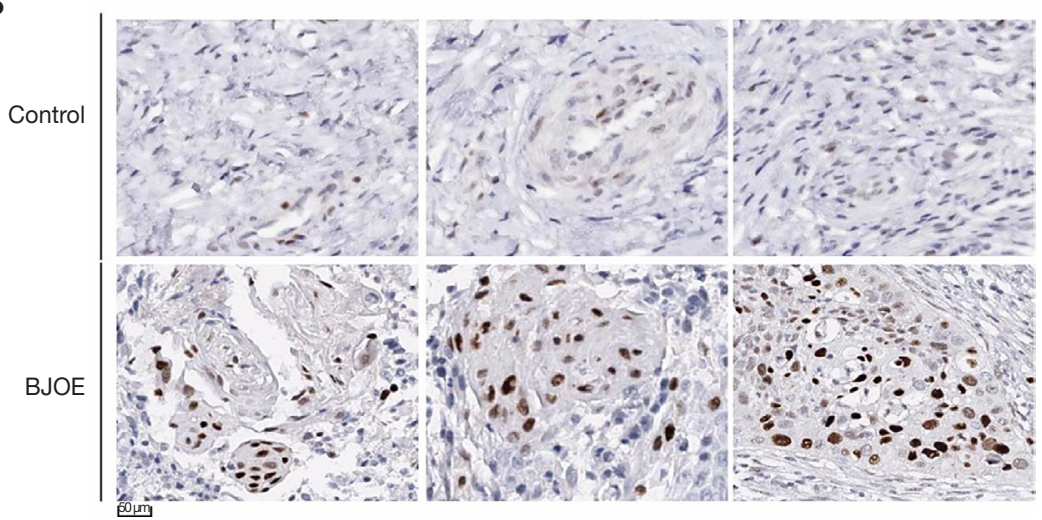

Figure 6 Effects of BJOE on p-ERK and p53 protein expression in the xenograft nude mouse model. Immunohistochemical staining images of (A) p-ERK expression and (B) p53 expression in representative tumor tissues from nude mice bearing SiHa cells after treatment with and without BJOE for 30 days, respectively. Scale bar: $50 \mu \mathrm{m}$. BOJE, Brucea javanica oil emulsion; ERK, extracellular-signal regulated kinase; p-ERK, phospho-ERK.

implicated in affecting drug sensitivity in tumors (32-34). Previous studies have indicated that suppression of ERK2 increases the resistance of cervical cancer cells to cisplatin through enhancing cisplatin-induced NF- $\kappa \mathrm{B}$ activation $(35,36)$. Brusatol, a bioactive component of BJOE, has been found to protect against ulcerative colitis via suppressing the $\mathrm{NF}-\mathrm{\kappa B}$ signaling pathway in a rat model (37). Inhibition of $\mathrm{NF}-\mathrm{\kappa B}$ translocation has been associated with the apoptotic effect of BJOE in colon cancer cells in vitro (38). Our results demonstrated that BJOE induced the phosphorylation of ERK and inhibited the expression of NF- $\mathrm{kB}$. These results, along with previous findings that BJOE improves the efficacy of chemotherapy in non-small cell lung cancer (11), suggest that BJOE possibly increases the chemotherapy sensitivity of cervical cancer cells via the ERK/MAPK and NF- $\kappa \mathrm{B}$ signaling pathways. Future studies are needed to test this hypothesis.

In this study, we explored the mechanisms driving BJOE- induced apoptosis and subsequent tumor suppression. Our results indicate that BJOE-induced apoptosis is partly dependent on the activation of caspase-3. Caspase- 3 is well recognized as a proapoptotic molecule in drug-induced apoptosis of tumor cells. However, increased activation of caspase- 3 has been detected in HPV16-expressing cells, despite downregulation of p53 (39). The HPV E6 and E7 oncoproteins have been found to activate rather than suppress activation of caspase- 3 to induce viral genome amplification upon differentiation (40). These seemingly paradoxical results indicate that caspase- 3 activity is dependent upon the cell type and stimuli and suggest the involvement of p53-independent apoptotic pathways in E6induced apoptosis. In the present study, using Western blot and IHC analyses, we identified a few targets for BJOEinduced apoptosis in SiHa cells (Figure 3), supporting the potential broad-spectrum antitumor effects of BJOE. 
However, it is not clear whether these signaling molecules modulated by BJOE to exhibit apoptotic effects also play a significant role in diverse cervical cancer cell lines. More studies are needed to evaluate the importance of these signaling molecules in cervical carcinogenesis.

The present study has several limitations. First, only one cervical cancer cell line, SiHa, was analyzed in vitro and in vivo. Therefore, whether the findings are generalizable to other cervical cancer cells are uncertain. Second, no systematic analysis was performed to evaluate the pharmacokinetic profile of BJOE. The optimal dose for safety and efficacy remains to be determined. Lastly, the present study only assessed several key signaling molecules related to apoptosis. More studies are needed to explore possible activities and mechanisms behind the antitumor effects of BJOE.

In conclusion, our results indicate that the tumorsuppressive effects of BJOE in HPV16-expressing $\mathrm{SiHa}$ cells are mediated through inhibition of E6 expression and induction of apoptosis, which occurs through the regulation of various signaling pathways. Our in vitro and in vivo results provide a compelling rationale for further research into the clinical use of BJOE alone or as an adjuvant to chemotherapeutic agents for cervical cancer treatment. Preclinical validation of BJOE use in various cervical cancer models as well as molecular biology research for better understanding of the underlying mechanisms would be an invaluable sequel to this study.

\section{Acknowledgments}

Funding: This work was supported by the Research Foundation of Medical Science and Technology of Guangdong Province (No. B2019132).

\section{Footnote}

Conflicts of Interest: All authors have completed the ICMJE uniform disclosure form (available at http://dx.doi. org/10.21037/tcr.2019.12.62). The authors have no conflicts of interest to declare.

Ethical Statement: The authors are accountable for all aspects of the work in ensuring that questions related to the accuracy or integrity of any part of the work are appropriately investigated and resolved. All animal experiments were conducted in accordance with the National Institutes of Health Guide for the Care and Use of Laboratory Animals, and the animal protocol was approved by the Ethics Committee of Jinan University (No. SYXY2017-0174).

Open Access Statement: This is an Open Access article distributed in accordance with the Creative Commons Attribution-NonCommercial-NoDerivs 4.0 International License (CC BY-NC-ND 4.0), which permits the noncommercial replication and distribution of the article with the strict proviso that no changes or edits are made and the original work is properly cited (including links to both the formal publication through the relevant DOI and the license). See: https://creativecommons.org/licenses/by-nc$\mathrm{nd} / 4.0 /$.

\section{References}

1. Bray F, Ferlay J, Soerjomataram I, et al. Global cancer statistics 2018: GLOBOCAN estimates of incidence and mortality worldwide for 36 cancers in 185 countries. CA Cancer J Clin 2018;68:394-424.

2. Ferlay J, Ervik M, Lam F, et al. Global Cancer Observatory: Cancer Today. Lyon, France: International Agency for Research on Cancer, 2018. Available online: https://gco.iarc.fr/today/home. Accessed June 24, 2019.

3. Lee SJ, Yang A, Wu TC, et al. Immunotherapy for human papillomavirus-associated disease and cervical cancer: review of clinical and translational research. J Gynecol Oncol 2016;27:e51.

4. Zhao Q, Modis Y, High K, et al. Disassembly and reassembly of human papillomavirus virus-like particles produces more virion-like antibody reactivity. Virol J 2012;9:52.

5. Hall MT, Simms KT, Lew JB, et al. The projected timeframe until cervical cancer elimination in Australia: a modelling study. Lancet Public Health 2019;4:e19-27.

6. Markowitz LE, Tsu V, Deeks SL, et al. Human papillomavirus vaccine introduction--the first five years. Vaccine 2012;30 Suppl 5:F139-48.

7. Yin Y. HPV vaccination in China needs to be more costeffective. Lancet 2017;390:1735-6.

8. Kaufmann AM, Backsch C, Schneider A, et al. HPV induced cervical carcinogenesis: molecular basis and vaccine development. Zentralbl Gynakol 2002;124:511-24.

9. Wright AA, Howitt BE, Myers AP, et al. Oncogenic mutations in cervical cancer: genomic differences between adenocarcinomas and squamous cell carcinomas of the cervix. Cancer 2013;119:3776-83.

10. He LJ, Luo HQ, Xiang L. Efficacy of brucea Javanica oil 
emulsion combined radiotherapy on treating advanced esophageal carcionma. Chinese Journal of Experimental Traditional Medical Formulae 2010;16:212-4.

11. Nie YL, Liu KX, Mao XY, et al. Effect of injection of brucea javanica oil emulsion plus chemoradiotherapy for lung cancer: a review of clinical evidence. J Evid Based Med 2012;5:216-25.

12. Liu J, Huang XE, Tian GY, et al. Phase II study on safety and efficacy of Yadanzi(R) (Javanica oil emulsion injection) combined with chemotherapy for patients with gastric cancer. Asian Pac J Cancer Prev 2013;14:2009-12.

13. Wang Q, Wang M, He X, et al. Meta-analysis on treatment of non-small cell lung cancer with brucea javanica oil emulsion in combination with platinumcontained first-line chemotherapy. Zhongguo Zhong Yao Za Zhi 2012;37:2022-9.

14. Jin W, Han H, Zhou S, et al. Therapeutic efficacy of brucea javanica oil emulsion (BJOE) combined with transcatheter hepatic arterial chemoembolization (TACE) in patients with primary liver cancer. Int J Clin Exp Med 2015;8:18954-62.

15. Zhou JP, Yang HX. Meta-analysis on efficacy and safety of Brucea javanica oil emulsion injection combined with chemotherapy for patients with advanced gastric carcinoma. Zhongguo Zhong Yao Za Zhi 2016;41:326-32.

16. Gao H, Lamusta J, Zhang WF, et al. Tumor Cell Selective Cytotoxicity and Apoptosis Induction by an Herbal Preparation from Brucea javanica. N Am J Med Sci (Boston) 2011;4:62-6.

17. Hassan M, Watari H, AbuAlmaaty A, et al. Apoptosis and molecular targeting therapy in cancer. Biomed Res Int 2014;2014:150845.

18. Singh S, Upadhyay AK, Ajay AK, et al. p53 regulates ERK activation in carboplatin induced apoptosis in cervical carcinoma: a novel target of p53 in apoptosis. FEBS Lett 2007;581:289-95.

19. Gkouveris I, Nikitakis N, Avgoustidis D, et al. ERK1/2, JNK and STAT3 activation and correlation with tumor differentiation in oral SCC. Histol Histopathol 2017;32:1065-76

20. James MA, Lee JH, Klingelhutz AJ. Human papillomavirus type 16 E6 activates NF-kappaB, induces cIAP-2 expression, and protects against apoptosis in a PDZ binding motif-dependent manner. J Virol 2006;80:5301-7.

21. Li J, Jia H, Xie L, et al. Association of constitutive nuclear factor-kappaB activation with aggressive aspects and poor prognosis in cervical cancer. Int J Gynecol Cancer 2009; 19:1421-6.
22. Pommier Y, Sordet O, Antony S, et al. Apoptosis defects and chemotherapy resistance: molecular interaction maps and networks. Oncogene 2004;23:2934-49.

23. Liu JJ, Ho JY, Lee HW, et al. Inhibition of Phosphatidylinositol 3-kinase (PI3K) Signaling Synergistically Potentiates Antitumor Efficacy of Paclitaxel and Overcomes Paclitaxel-Mediated Resistance in Cervical Cancer. Int J Mol Sci 2019. doi: 10.3390/ijms20143383.

24. Fuhong D, Xiang G, Haiying L, et al. Evaluation of efficacy and safety for Brucea javanica oil emulsion in the control of the malignant pleural effusions via thoracic perfusion. BMC Cancer 2018;18:411.

25. Xie JH, Lai ZQ, Zheng XH, et al. Apoptosis induced by bruceine D in human non-small-cell lung cancer cells involves mitochondrial ROS-mediated death signaling. Int J Mol Med 2019;44:2015-26.

26. Qiu ZH, Zhang WW, Zhang $\mathrm{HH}$, et al. Brucea javanica oil emulsion improves the effect of radiotherapy on esophageal cancer cells by inhibiting cyclin D1-CDK4/6 axis. World J Gastroenterol 2019;25:2463-72.

27. Jung JI, Kim SY, Park KY, et al. In vitro combinatorial anti-proliferative and immunosuppressive effects of Brucea javanica extract with CX-4945 and imatinib in human T-cell acute lymphoblastic leukemia cells. Biomed Pharmacother 2018;106:403-10.

28. Lai ZQ, Ip SP, Liao HJ, et al. Brucein D, a Naturally Occurring Tetracyclic Triterpene Quassinoid, Induces Apoptosis in Pancreatic Cancer through ROS-Associated PI3K/Akt Signaling Pathway. Front Pharmacol 2017;8:936.

29. Jiang L, Wang W, He Q, et al. Oleic acid induces apoptosis and autophagy in the treatment of Tongue Squamous cell carcinomas. Sci Rep 2017;7:11277.

30. Narisawa-Saito M, Kiyono T. Basic mechanisms of highrisk human papillomavirus-induced carcinogenesis: roles of E6 and E7 proteins. Cancer Sci 2007;98:1505-11.

31. Koivusalo R, Mialon A, Pitkanen H, et al. Activation of p53 in cervical cancer cells by human papillomavirus E6 RNA interference is transient, but can be sustained by inhibiting endogenous nuclear export-dependent p53 antagonists. Cancer Res 2006;66:11817-24.

32. Baldwin AS. Control of oncogenesis and cancer therapy resistance by the transcription factor NF-kappaB. J Clin Invest 2001;107:241-6.

33. Hill CS, Treisman R. Transcriptional regulation by extracellular signals: mechanisms and specificity. Cell 1995;80:199-211.

34. Abrams SL, Steelman LS, Shelton JG, et al. The Raf/ 
MEK/ERK pathway can govern drug resistance, apoptosis and sensitivity to targeted therapy. Cell Cycle 2010;9:1781-91.

35. Yeh PY, Chuang SE, Yeh KH, et al. Increase of the resistance of human cervical carcinoma cells to cisplatin by inhibition of the MEK to ERK signaling pathway partly via enhancement of anticancer drug-induced NF kappa B activation. Biochem Pharmacol 2002;63:1423-30.

36. Yeh PY, Yeh KH, Chuang SE, et al. Suppression of MEK/ ERK signaling pathway enhances cisplatin-induced NFkappaB activation by protein phosphatase 4-mediated NF-kappaB p65 Thr dephosphorylation. J Biol Chem 2004;279:26143-8.

37. Zhou J, Wang T, Dou Y, et al. Brusatol ameliorates 2, 4, 6-trinitrobenzenesulfonic acid-induced experimental colitis in rats: Involvement of NF-kappaB pathway and NLRP3 inflammasome. Int Immunopharmacol 2018;64:264-74.

38. Bagheri E, Hajiaghaalipour F, Nyamathulla S, et al. The apoptotic effects of Brucea javanica fruit extract against HT29 cells associated with p53 upregulation and inhibition of NF-kappaB translocation. Drug Des Devel Ther 2018;12:657-71.

39. Gray LJ, Bjelogrlic P, Appleyard VC, et al. Selective induction of apoptosis by leptomycin B in keratinocytes expressing HPV oncogenes. Int J Cancer 2007;120:2317-24.

40. Moody CA, Fradet-Turcotte A, Archambault J, et al. Human papillomaviruses activate caspases upon epithelial differentiation to induce viral genome amplification. Proc Natl Acad Sci U S A 2007;104:19541-6.
Cite this article as: Ye L, Zhao JF, Wang YM, Chen WH, Qian $\mathrm{S}$, Zhou ZG, Xu M. Brucea javanica oil emulsion suppresses tumor growth in human cervical cancer cells through inhibition of the E6 oncogene and induction of apoptosis. Transl Cancer Res 2020;9(2):918-929. doi: 10.21037/tcr.2019.12.62 